\title{
Eğitim Amaçlı Sanal Gerçeklik Uygulamalarında Kullanılan Cihazların Daldırma Açısından İncelenmesi ${ }^{* * \dagger}$ Araştırma Makalesi/Research Article
}

\author{
Dillkim Ecem EMRE ${ }^{1}$ (D) Murat SELÇUK ${ }^{2}$, (D) Veli Özcan BUDAK ${ }^{3}$, (D) Mehmet BÜTÜN² ${ }^{2}$ (D) İrfan \\ ŞİMŞEK ${ }^{4}$ \\ 1 İşletme Enformatiği Bölümü, İșletme Fakültesi, Marmara Üniversitesi, İstanbul, Türkiye \\ ${ }^{2}$ Enformatik Bölümü, İstanbul Üniversitesi, İstanbul, Türkiye \\ ${ }^{3}$ Bilgi İşlem Daire Başkanlığı, Kırklareli Üniversitesi, Kırklareli, Türkiye \\ ${ }^{4}$ Bilgisayar ve Öğretim Teknolojileri Eğitimi Bölümü, Hasan Ali Yücel Eğitim Fakültesi, İstanbul Üniversitesi - Cerrahpaşa, İstanbul, Türkiye

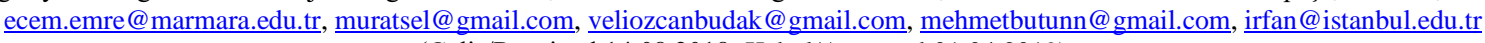 \\ (Geliş/Received:14.08.2018; Kabul/Accepted:01.04.2019)
}

DOI: $10.17671 /$ gazibtd.453381

\begin{abstract}
$\ddot{O}_{z} \boldsymbol{e t}$ - Teknolojide yaşanan gelişmeler, farklı araç ve yöntemlerin eğitim amaçlı kullanımına olanak sağlamaktadır. İlgili alanyazın incelendiğinde, son yıllarda giderek artan kullanım oranlarıyla sanal gerçeklik (SG) teknolojisinin eğitim amaçlı çalışmalarda sıklıkla tercih edilmeye başlandığı görülmektedir. SG, bireyin farklı duyu organlarını uyararak fiziki bir ortamdan sanal bir ortama geçişine olanak sağlayan bir teknolojidir. Bu çalışmada; SG uygulamalarıyla eğitim amaçlı gerçekleştirilen çalışmalarda kullanılan cihazlar, daldırma (bireyin sanal gerçeklik cihazlarıyla fiziki ortamla olan bağının belirli sınırlılıklarla kesilerek sanal ortamda bulunma hissi) etkileri yönünden ele alınmış, yeterlilikleri ve sundukları fırsatlar bakımından incelenmiştir. Scopus veri tabanında 2013-2017 yılları arasında yayımlanmış, "sanal gerçeklik (virtual reality)" kelime grubunu başlık ve "eğitim (education)" kelimesini başlık, özet ve anahtar kelime olarak barındıran ve daldırma etkisi sunan cihazları içeren, 30 çalışma içerik analizi yöntemi ile belirlenen kısıtlar çerçevesinde incelenerek araştırma gerçekleştirilmiştir. İncelenen çalışmaların tamamında (\%100) görme duyusuna hitap eden cihaz(lar)ın kullanıldı ğı, \%50'sinde işitme ve \%47'sinde dokunma duyusuyla etkileşim içeren cihaz(lar)ın kullanıldığı görülmüştür. Koku duyusuna hitap eden çalışmaya ise rastlanmamıştır. Araştırmaya dahil edilen çalışmaların \%43'ünde tam daldıııı SG uygulamalarının, \%53'ünde ise yarı daldırıcı SG uygulamalarının kullanıldığı tespit edilmiştir. Kullanılan sistem tipi bakımından çalışmaların \%66'sında SG gözlüklerinin (Head Mounted Display-HMD) tercih edildiği, \%10'unda ise sanal mağaraların (CAVE) kullanıldığı görülmüştür. Araştırma kapsamında yapılan analizler sonucunda tespit edilen olan tüm bulgular detaylı bir şekilde aktarılmıştır. Bu çalışmanın, eğitim alanında SG ile ilgili çalışmalar yapan araştırmacılar için rehber niteliği taşıyan bir kaynak olacağı düşünülmektedir.
\end{abstract}

Anahtar Kelimeler — sanal gerçeklik, eğitimde sanal gerçeklik, daldırma

\section{Investigation of Devices Used in Virtual Reality Applications For Education Purposes in Terms of Immersion}

\footnotetext{
Abstract - Technological developments enable the use of different tools and methods for educational purposes. When the relevant literature is examined, it is seen that virtual reality (VR) technology is becoming more and more preferred for educational purposes with increasing usage rates in recent years. VR is a technology that allows individuals to pass from a physical environment to a virtual environment by stimulating different sensory organs. In this study, the devices

* Bu çalışma, İstanbul Üniversitesi Bilimsel Araştırma Projeleri Birimi tarafından, 08.08.2018 tamamlanmış olan SDP-2017-22377 kodlu proje ile desteklenmiştir.

$\dagger$ Bu çalışma, 3-5 Mayıs 2018 tarihleri arasında Alanya'da gerçekleştirilen 4. Uluslararası Eğitim Bilimleri Sempozyumu'nda sunulan "Eğitim Amaçlı Sanal Gerçeklik Uygulamalarında Kullanılan Cihazların Daldırma Açısından İncelenmesi” başlıklı bildirinin genişletilmiş halidir.
} 
used in the studies conducted for educational purposes with VR applications, their features and opportunities were examined in terms of their immersion effects (creating the feeling of being in the virtual environment by cutting the connection of individual with the physical environment with certain limitations). In the scope of the study, 30 papers published in the Scopus database between the years 2013-2017, which include devices that have immersion effect and containing the words "virtual reality" in title and "education" in title, abstract and keywords fields were analysed with content analysis. It is seen that, in all of the studies visual sense, in 50\% sense of hearing and in $47 \%$ sense of touch was addressed with the devices. Study that addressed the sense of smell is not found. It was determined that $43 \%$ of the studies included in the study had full immersion SG applications and 53\% had semi-submerged SG applications. In terms of the used system type, VR glasses (head mounted display-HMD) was preferred in 66\% of the studies and virtual caves (cave automatic virtual environment - CAVE) were used in 10\%. All the findings of the analyses were given in detail. This study is thought to be a guide resource for researchers working on VR in the field of education.

Keywords - virtual reality, virtual reality in education, immersion

\section{GİRIŞ (INTRODUCTION)}

Teknolojik gelişmeler günlük hayatımızı pratikleştirmenin yanı sıra farklı türde deneyimlerin oluşmasına da olanak sağlamaktadır. Bu gelişmelerden biri olan sanal gerçeklik (SG) teknolojisi, birbirinden benzersiz alanlardan yararlanılabilen ve ihtiyaca özgü olarak esnetilebilen bir yapıya sahiptir. Aynı zamanda, duyularımızda oluşturduğu gerçeklik hissi sayesinde belki de daha önce deneyimleyemediğimiz birçok etkileşimin oluşmasına olanak tanımaktadır. Guttentag [1], SG'yi bir kullanıcının yönlendirebileceği ve etkileşime girebileceği, bilgisayar tarafından oluşturulan üç boyutlu bir ortamın kullanımı olarak tanımlamıştır. Başka bir çalışmada ise SG, bilgisayarlar tarafindan oluşturulmuş, gerçeklik hissi ve yapay zeka ürünleriyle birlikte bilgi araçlarının entegrasyonunu barındıran ortamlarda, kullanıcılar arasında işbirliği ve etkileşim içeren bir teknoloji olarak tanımlanmıştır [2]. SG teknolojisi; mekânsal çoklu duyu gösterimine, etkileşime ve uzakta bulunma hissine odaklanır; gerçek zamanlı simülasyon tekniklerini ve sanal ortam yönetimini içerir [3]. Kısacası SG, çeşitli duyu organlarımızın uyarılmasıyla birlikte fiziki bir ortamdan sanal bir ortama geçişe olanak sağlayan oldukça etkileyici bir teknolojidir. Ortamlar arasındaki bu geçiş SG uygulamaları ve bu uygulamaların çalıştırıldığ ç̧eşitli cihazlar vasıtasıyla gerçekleştirilmektedir. Bu bağlamda bakıldığında eğitimde SG kullanımı birçok avantaj sağlamaktadır [4]. SG uygulamalarının gerçek ve sanal ortam arasında köprü vazifesi gördüğü söylenebilir. SG, bu çalışmanın odağında olan, eğitim alanında ise sıklıkla yararlanılan bir teknolojidir. $\mathrm{Bu}$ teknolojinin farklı öğrenme stillerinin tercih edildiği ve yaratıcı öğrenmenin gelişiminin desteklendiği durumlarda avantaj sağladığ belirtilmiştir [5]. SG deneyiminin yaşatılabilmesi için sanal gerçeklik gözlükleri (head mounted display - HMD) ve sanal mağaralar (Cave automatic virtual environment CAVE) gibi farklı teknolojilerden yararlanılmaktadır. Anthes vd. [6] yaptıkları çalışmada SG donanımları ile ilgili aşağıdaki gibi bir taksonomi oluşturmuştur. Buna göre SG cihazlarını, girdi ve çıktı cihazları olarak ikiye başlıkta ele almak mümkündür:

- Girdi cihazları: kontrol cihazları, yönlendirme cihazları, takip cihazları (Şekil 1)
- Çıktı cihazları: görsel, dokunma, birden fazla duyuya hitap eden cihazlar (Şekil 2)

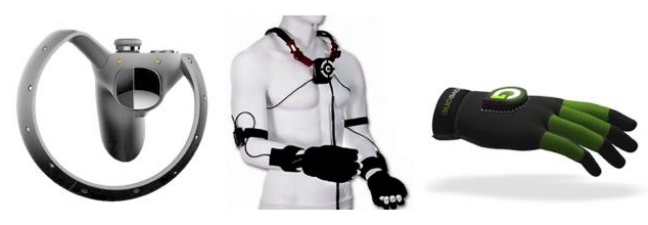

Şekil 1. Girdi cihazlarından joystick, vücut takip cihazı, eldiven [6].

(Input devices - joystick, posture and gesture tracking system, data glove)

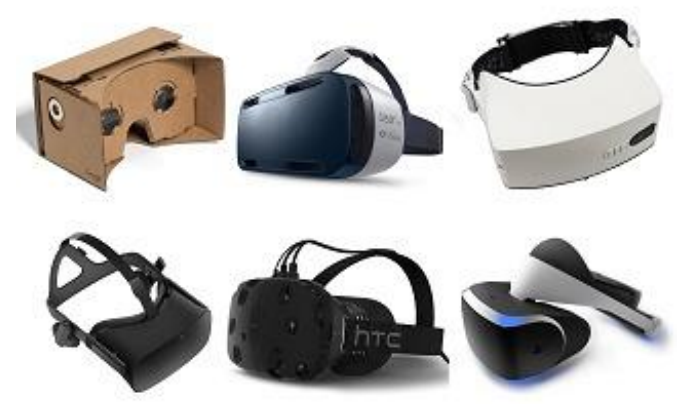

Şekil 2. Çıktı cihazlarından sanal gerçeklik gözlükleri [6]. (Output devices - HMD)

İlgili alanyazın incelendiğinde, SG teknolojisinin birçok alanda eğitim amaçlı kullanıldığı görülmektedir. Mimarlık, tarih, fen, matematik, tıp, askeriye ve havayolu endüstrisi gibi alanlarda eğitim amaçlı olarak bu teknolojinin kullanılabildiği aktarılmıştır [7]. Bunların yanı sıra; turizm, denizcilik, psikoloji, mimari gibi farklı alanlarda SG'nin kullanıldığı çeşitli çalışmaların olduğu görülmektedir [8]-[15]. Sağlık alanında ise alanyazın derlemesi olarak birçok çalışmada SG teknolojilerinin irdelendiği tespit edilmiştir [16]-[32]. Ayrıca alanyazında yer alan bazı deneysel çalışmalarda SG teknolojilerinin; tıp [33]-[36], yabanc1 dil [37], madencilik [38], yaya güvenliği [39], müzik [40] ve takım çalışması [41] ile ilgili eğitimlerde kullanıldığı gözlemlenmiştir. Yapılmış olan farklı türlerdeki bu çalışmalarda ortak amacın; SG teknolojisini eğitim alanında eksik görülen noktaların giderilmesi ve/veya var olan bir sürecin daha etkin hale getirilmesi için kullanılması olduğu görülmektedir. 
SG teknolojisinde yaşanan son gelişmeler, SG uygulamalarında kullanılan cihazların gelişmesine, düşen maliyetler sayesinde kolay erişilebilir olmasına ve bu teknolojinin daha yaygın kullanımına imkân tanımıştır. Yaşanan teknolojik gelişmeler, eğitim alanında yapılan çalışmalarda kullanılan SG cihazlarının daldırma (immersion) etkilerinin de gelişmesini sağlamıştır. Daldırma, sunulan sanal dünyada olma yanılsaması olarak düşünülebilmekte ve kullanıcıyı ses ve görüntüyle kuşatarak gerçekten orada olduğuna inandırabilmektedir [42]. SG teknolojilerinde daldırma; kişinin gerçek dünyaya benzeyen sanal bir ortama yerleştirilmesi, bu dünyanın kendini çevrelediğini hissetmesi, nesnelerle etkileşime girmesi, nesnelerin gerçek dünyadaki gibi sıradan bir konuma sahip olması ve keşfedilebilir olması gibi etkiler ile açıklanabilir [43].

SG ortamlarında gerçekleştirilen becerileri eğitimlerinde daldırma ve uzakta var olma hissi düzeyleri anahtar faktörler arasında yer alır, bu nedenle SG ortamları daldırma açısından yüksek seviyeye sahip olacak şekilde gerçekçi bir biçimde tasarlanmalıdır [45].

SG ortamlarını görsel açıdan tam daldırıcı SG ortamları (HMD kullanılanlar), yarı daldırıcı $S G$ ortamları (büyük projeksiyon ekranları kullananlar) ve daldırıcı olmayan $S G$ ortamlarl (bilgisayar ekranı kullananlar) olmak üzere üçe ayıran Gutierrez vd. [46], günümüzde SG sistemlerinin görme, işitme ve dokunma duyularına hitap edilmesi süreçlerini içerdiğini, teknolojinin karmaşıklığı nedeniyle koku ve tat alma duyularına hitap etmenin sürece dahil edilemediğini belirtmektedir. MagnenatThalmann ve Bonanni [48] ise SG uygulamalarında dokunsal cihazların kullanımını ele aldığı çalışmasında, birden fazla duyuya hitap edilen sanal gerçeklik uygulamalarının daldırma açısından daha sürükleyici olduğunun altını çizerek, SG ortamlarının sadece görsel ve işitsel uyarıcıları içermesinin önemli bir sınırlılık olacağını belirtmektedir.

HMD, dokunma olanağı tanıyan eldivenler ve izleme cihazları gibi bileşenleri ile sanal ortamın bir parçası olma hissi veren SG ortamları, kullanıcılara en üst seviyede daldırma olanağı tanımaktadır [47]. Daldırıcı bir SG deneyiminin, görsel, işitsel ya da dokunsal uyarıcıların kullanımına bağlı olduğunu belirten Lecuyer [49] gereğinden fazla duyunun uyarıldığı SG ortamlarında yüksek bir daldırma sağlansa bile uyarıcıların doğru seviyelendirilmemesi gibi durumlarda uzakta var olma hissinin olumsuz yönde etkilenebileceğine dikkat çekmiştir.

SG, daldirıcı ortamlar sayesinde bireysel deneyimler sunarak, bilginin katılımcı tarafından aracı olmaksızın yapılandırılmasına olanak tanımaktadır [50]. Daldırıcı SG deneyimleri nesnelerin yakından incelenmesine olanak tanıyabilmekte ve gerçek hayatta deneyimlenmesi imkânsız olan durumlar için çeşitli firsatlar sunabilmektedir [51]. Nitekim Guttierez vd. [52] yüksek daldırma seviyesine sahip cihazlarla eğitim gören tıp öğrencilerinin, düşük daldırma seviyesine sahip cihazlarla eğitim görenlerden daha başarılı olduklarını tespit etmiştir. Jackson ve Fagan [53], iyi tasarlanmış ve daldırma etkisi yüksek SG ortamlarında hiçbir yöntemle ulaşılamayacak kadar faydalı eğitim deneyimleri yaşanabileceğini belirtmiştir. Bowman ve McMahan [54] ise yüksek seviyeli daldırma etkisinin etkileşim görevlerinde performans artışı sağladığını, uzamsal anlaşılırlığı (presence) arttırdığını ve bilgi dağınıklığını azalttığını tespit etmiştir. Alanyazında yer alan çalışmalar ışığında, eğitim alanında kullanılan SG teknolojilerinde daldırma etkisinin;

- $\quad$ öğrenme deneyimlerini pozitif yönde etkileyerek öğrencilerin performanslarını geliştirebileceği [53],

- uzamsal anlaşılırlığı arttırabileceği [54],

- bilgi dağınıklıklarını ortadan kaldırabileceği [52],[54],

- bireysel deneyimlere olanak tanıyarak yaparak, yaşayarak öğrenme ve bilginin doğrudan yapılandırılması gibi firsatlar sunabileceği [50],

söylenebilir. Yine alanyazında yer alan çalışmalar SG uygulamalarında daldırma etkisinin seviyesinin de oldukça önemli olduğunu göstermiştir [4],[50]. Bu nedenle eğitim alanında yapılacak çalışmalarda, kullanılan SG teknolojilerinin daldırma düzeyi hangi seviyede olduğu göz önünde bulundurulmalıdır. Dolayısıyla, daldırma düzeyi açısından, kullanılacak SG cihazlarının seçiminin, çalışmanın başarısını etkileyebileceği dikkate alınarak yapılmalıdır.

Hoffman vd. [44], daldırma ve uzakta var olma hissi açısından SG teknolojilerini düşük ve yüksek teknolojili SG sistemleri olmak üzere iki ayırmış ve yüksek teknolojili SG sistemlerinin; birden fazla duyuya hitap etme, yüksek çözünürlük, kullanıcının sanal dünya ile etkileşime girmesine izin verme, sınırlı görüş alanı yerine panaromik görüş alanı sunma gibi özellikleri açısından farklılaştığını belirtmiştir. $\mathrm{Bu}$ çalışmada, Hoffman vd. [44] ve Gutierrez vd. [46] tarafindan yapilan sinıflandirmalar göz önünde bulundurularak, araştırma kapsamında incelenen çalışmalardaki SG uygulamaları şu şekilde sınıflandırılacaktır:

- Birden fazla duyuya hitap eden, HMD'ler ve dokunsal ekipmanlarla kullanıcıya sanal dünya ile etkileşime girme olanağı tanıyan SG uygulamaları tam daldırıcı (fully immersive)

- Tek bir duyuya hitap ederek kullaniciya sadece HMD'ler aracılığıyla sanal dünya ile etkileşim olanağı tanımayan SG uygulamaları yarı daldirıcı (semi-immersive)

- HMD kullanılmayan SG uygulamaları daldırıc olmayan (non-immersive)

$\mathrm{Bu}$ araştırmanın amacı, eğitim alanında SG uygulamalarının kullanıldı ̆̆ı çalışmaların incelenmesi yoluyla, bu çalışmalarda kullanılan SG uygulamalarının daldırma düzeylerinin tespit edilmesidir. SG 
uygulamalarının daldırma düzeyi, sanal ortamda kullanıcının gerçek yaşamdakine ne ölçüde benzer bir deneyim yaşadığıyla doğrudan ilişkilidir. $\mathrm{Bu}$ nedenle kullanılan SG sistemleri ve cihazları daldırma düzeyini doğrudan etkileme potansiyeline sahiptir [55]. Nitekim kullanıcının gerçek yaşamdaki gibi gezinmesi, hareketlerinin sanal dünyaya aktarılması ve duyuları aracılığıyla sanal ortam ile etkileşim kurması kullanılan cihazların sunduğu olanaklara bağlıdır. $\mathrm{Bu}$ doğrultuda aşağıdaki hedeflere yönelik incelemeler gerçekleştirilmiştir:

- SG uygulamalarının kullanıldığı çalışma alanlarının ve ilgili daldırma düzeylerinin belirlenmesi

- Kullanılan SG sistem tiplerinin belirlenmesi

- Kullanılan SG cihazlarının belirlenmesi

- SG uygulamalarında hareket izleme sensörlerinin kullanım durumlarının belirlenmesi

- SG uygulamalarında kullanıcıların serbest hareket imkânı olup olmadığının belirlenmesi

- Kullanılan SG cihazlarının hangi duyu organlarına hitap ettiğinin belirlenmesi

$\mathrm{Bu}$ çalışmanın, eğitim alanında SG ile ilgili çalışmalar yapan ya da yapmayı düşünen araştırmacılar için rehber niteliği taşıyacağı düşünülmektedir.

\section{METOT (METHOD)}

\subsection{Araştırma Deseni (Research Design)}

$\mathrm{Bu}$ çalışma içerik analizi temel alınarak gerçekleştirilmiştir. İçerik analizin; metinlerin düzenlenmesi, sınıflandırılması, karşılaştırılması ve metinlerden teorik sonuçlar çıkarılmasından oluşan bir araştırma tekniğidir [56]. Bu çalışmada içerik analizi, birbirlerine benzeyen verileri belirli kavramlar ve temalar çerçevesinde bir araya getirerek okuyucunun anlayacağ biçime dönüştürmeye olanak tanımasından dolayı [57],[58] tercih edilmiştir. İçerik analizi yöntemi ile ele alınan eğitim alanında SG uygulaması kullanılan çalışmalar, kullanılan SG cihazlarının daldırma etkileri açısından incelenmiştir.

\subsection{Verilerin Toplanması (Data Collection)}

Çalışma kapsamında yapılan sistematik derleme; Scopus veri tabanı üzerinde bulunan, 2013-2017 y1lları arasında yayımlanan, "sanal gerçeklik (virtual reality)" anahtar kelime grubunu başlık bölümünde ve "eğitim (education)" anahtar kelimesini başlık, özet veya anahtar kelime bölümlerinde içeren makaleleri kapsamaktadır.

Çalışmaların araştırma kapsamına dâhil edilme kriterleri;

- makale olarak yayınlanmış çalışmalar,

- makale dili İngilizce olan çalışmalar,

- daldırma etkisi olan (yarı daldırıcı ve tam daldırıcı) SG odaklı çalışmalar olarak belirlenmiştir.

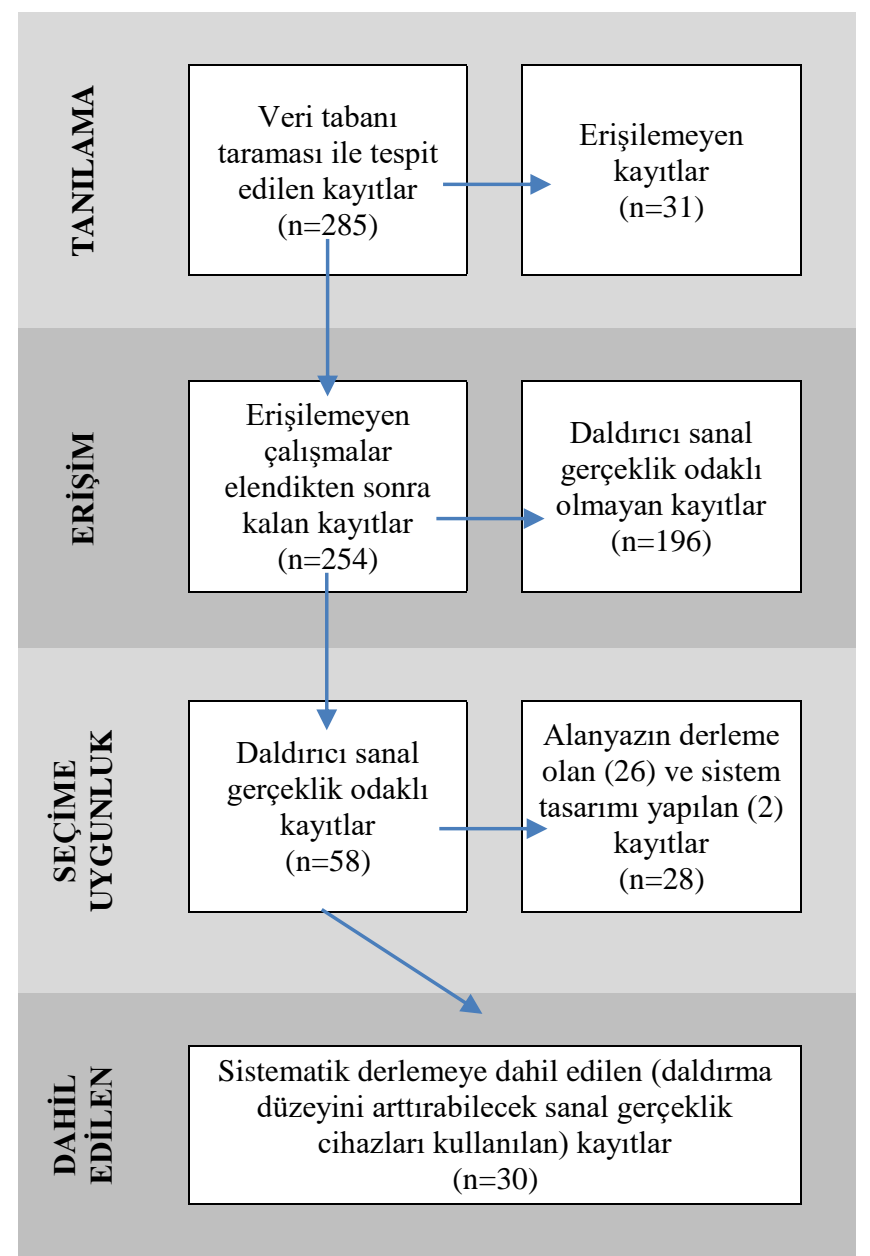

Şekil 3. Makale seçim süreci

(Paper selection process)

Arama sonuçlarına göre 285 çalışma listelenmiş, erişilemeyen çalışmalar elenerek, 254 çalışma incelenmiştir. Ön değerlendirmeler sonucunda 196 çalışmanın daldırıcı olmayan (non-immersive) SG odaklı çalışmalar oldukları tespit edilerek 58 çalışma ele alınmıştır. Yapılan analizler sonucunda 26 çalışmanın alanyazın derleme olduğu, 2 çalışmada sistem tasarımı yapıldığı, 30 çalışmada ise daldırma düzeyini arttırabilecek SG cihazlarının kullanıldığı görülmüştür. Makalelerin seçim süreci Şekil 3'te gösterilmiştir.

\subsection{Veri Analizi (Data Analysis)}

Çalışma kapsamında makaleler içerik analizi ile incelenmiştir. Analiz sonuçları istatistiki yöntemler (yüzde değerleri) ile elde edilmiş; incelenen farklı özellikler grafik ve tablolar halinde sunulmuştur.

\section{BULGULAR (FINDINGS)}

Çalışma kapsamında analiz edilen 30 çalışmaya ait; künye bilgileri, araştırma alanı (konu), daldırma düzeyi, duyulara (görme, işitme, dokunma, koklama) hitap etme, katılımcının serbest hareket etmesine olanak tanıma, SG 
sistem tipi, kullanılan cihaz, hareket izleme sensörü kullanımı ve akıllı telefon kullanımı ile ilgili veriler toplu olarak Error! Reference source not found.'de verilmiştir.

\section{1. Çalışmaların Yıllara Göre Dağılımı (Distribution of Studies by Years)}

Bu çalışma kapsamında 2013 ve 2017 yılları arasında incelenen çalışmaların yıllara göre dağılımı Şekil 4'teki gibidir. $\mathrm{Bu}$ dağılım incelendiğine; daldırma düzeyini arttırabilecek cihazların kullanıldığı SG çalışmalarının sayısında son yıllarda bir artış olduğu görünmektedir.

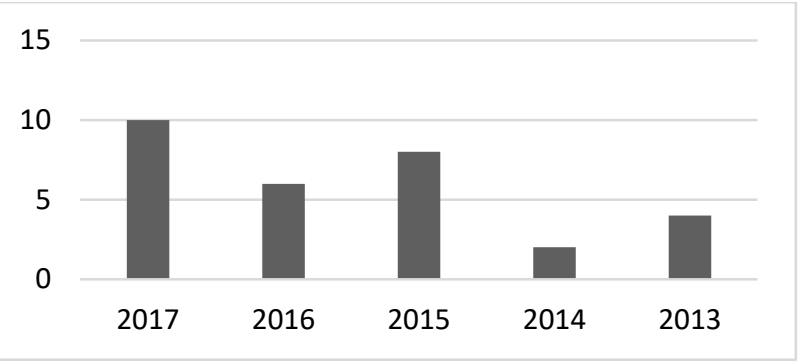

Şekil 4. Yıllara Göre Çalışma Sayısı (Number of studies by years)

\section{2. Çalışmaların Konulara ve Daldırma Düzeylerine Göre Dă̆llımı (Distribution of Studies by Topics)}

Araştırmaya dâhil edilen çalışmaların konulara göre dağılımı incelendiğinde çalışmaların yaklaşık yarısının (16 çalışma - \%53) sağlık alanında yapılan SG eğitim çalışmaları olduğu görülmektedir (Error! Reference source not found.). SG uygulamalarının; kimya eğitimi, mühendislik eğitimi, mekânsal-uzamsal eğitim ve bilişsel eğitim gibi çeşitli alanlardaki eğitim faaliyetlerinde kullanıldığı, ayrıca müzik becerileri, iletişim becerileri ve motor becerileri gibi çeşitli becerilerin geliştirilmesinde de kullanıldığı görülmektedir (Error! Reference source not found.).

Daldırıcı olmayan SG uygulamalarının araştırma kapsamı dışında bırakıldığı göz önünde bulundurularak, araştırmaya kapsamındaki çalışmalar daldırma düzeyleri açısından değerlendirildiğinde; tam daldırıcı SG uygulaması kullanılan çalışma sayısının (13 çalışma \%43), yarı daldırıcı SG uygulaması kullanılan çalıșma sayısından (17 çalışma - \%57) düşük olduğu görülmektedir (Error! Reference source not found.).

İncelenen çalışmaların konulara göre daldırma düzeylerine bakıldığında ise, özellikle sağlık alanında cerrahi ile ilgili gerçekleştirilen 10 çalışmanın büyük bir bölümünde (9 çalışma - \%90) birden fazla duyuya hitap eden tam daldırıcı $S G$ uygulamalarının kullanıldı ğ görülmektedir (Error! Reference source not found.).
SG sistem tipi; kullanıcıların farklı duyularına hitap etme, serbest hareket imkânı tanıma ve sanal dünyaya etkileşime olanak tanıma gibi yönlerden, daldırma düzeyini belirleyen en önemli faktörlerden biri olarak değerlendirilebilir. Incelenen 30 çalışma arasından 20 çalışmada HMD (\%67) kullanıldığ 1,3 çalışmada CAVE ve HMD'lerin birlikte (\%10) kullanıldı ̆̆ı, 3 çalışmada dV-Trainer (\%10) ve 4 çalışmada ise da Vinci Skills Simulator (\%13) kullanıldığ 1 görülmüştür.

dV-Trainer ve da Vinci Skills Simulator SG sistemleri cerrahi eğitimlerinde kullanılan ve tıp alanında öğrencilere sanal bir operasyon yapma olanağı tanıyan sistemlerdir [72],[73]. Her iki sistemde de dokunma yoluyla sanal dünyayla etkileşim imkânı tanınırken, serbest gezinme olanağı bulunmamaktadır. Cerrahi operasyonlarda serbest gezinme imkânının gerçek durumlarda da fazla yaşanmadığı ve cerrahın operasyon boyunca bir odak üzerinde çalıştığı düşünüldüğünde, bu sistemlerin tam daldırıcı $S G$ sistemleri oldukları söylenebilir.

CAVE ve HMD kullanılan SG sistem tiplerinde ise, sistemin daldırma düzeyini kullanılan cihazların sunduğu olanaklar ve bu olanakların ne ölçüde kullanıldığı belirlemektedir.

\section{4. Çalıșmalarda Kullanılan SG Cihazlarının Çeșitleri (Types of VR Devices)}

Araştırma kapsamında incelenen çalışmalarda en sık kullanılan SG cihazının Oculus Rift cihazı olduğu görülmüştür (8 çalışma - \%27). Çalışmaların bazılarında kullanılan cihazın açıkça belirtilmediği tespit edilmiştir. Bazı çalışmalarda birden fazla cihaz kullanımı tercih edildiği, ancak çalışmaların büyük bir çoğunluğunda tek bir SG cihazından yararlanıldığı görülmüştür.

Çalışmalarda kullanılan SG cihazları daldırma seviyesi açısından değerlendirildiğinde; HMD'lerin ses ve görüntü duyularına hitap ederek kullanıcılara serbest hareket olanağı tanıdığı, ayrıca Oculus Rift ve Samsung Gear VR gibi HMD'lerin kullanıcılara dokunma yoluyla sanal dünyalarla etkileşim kurma olanağı tanımasına rağmen bazı çalışmalarda bu özelliklerin kullanılmadığı tespit edilmiștir (Tablo 2).

\subsection{Akıllı Telefon Kullanımı (Smart Phone Usage)}

Analiz edilen 30 çalışma arasından 5 çalışmada (\%17) akıllı telefon ile çalışan SG uygulamaları kullanıldığı, 16 çalışmada ise (\%53) akıllı telefon olmadan çalışan SG uygulamaları kullanıldığı görülmektedir. Bunun yanı sıra 9 çalışmada (\%30) akıllı telefon ile çalışan SG cihazları kullanım durumu hakkında bilgi verilmemiştir. Akıllı telefon kullanılan çalışmaların son yıllarda artış gösterdiği; 4'ünün 2017 yılında, 1'inin ise 2016 yılında yapıldı̆̆ 1 tespit edilmiştir. 
3.6. Hareket İzleme Sensörü Kullanımı (Motion Tracking Sensor Usage)

İncelenen 30 çalışma arasından 16 çalışmada (\%53) hareket izleme sensörü kullanıldığı, 14'ünde (\%47) ise katılımciların hareketlerinin sistem tarafindan takip edilmediği görülmüştür. Hareket izleme sensörleri kullanıciların hareketleri hakkında veri toplayarak ve bu hareketleri sanal ortama transfer ederek daldırma düzeyini arttırabilecek bir potansiyele sahiptir.

\subsection{Serbest Hareket Imkânı (Free Movement Opportunity)}

Analiz edilen 30 çalışmanın sadece 12'sinde (\%40) kullanıcılara uygulama esnasında serbest hareket imkânı verilmiştir. 13 çalışmada (\%43) serbest hareket imkânı bulunmamaktadır. Bunun yanı sıra 5 çalışmada (\%17) kullaniciların uygulama esnasinda serbest hareket imkânına dair bilgi verilmemiştir. Kullanıcıların cihazlara bağlı olmadan serbestçe hareket edebilmeleri daldırma düzeyini arttırarak SG deneyimlerinin iyileşmesine olanak sağlamaktadır.

\subsection{Duyulara Hitap Etme (Addressing the Senses)}

Araştırmaya dâhil edilen çalışmalar duyulara hitap etmeleri açısından değerlendirildiğinde; tamamında görme duyusuna hitap edildiği, 15 çalışmada (\%50) işitme duyusuna, 14 çalışmada $(\% 47)$ ise dokunma duyusuna hitap edildiği tespit edilmiştir. Koklama duyusuna hitap edilen çalışma bulunmamaktadır. Sadece 5 çalıșmada (\%17) işitme, dokunma ve görme duyularına birlikte hitap edildiği tespit edilmiştir. Daha fazla duyuya hitap edilmesi SG deneyiminin daldırma etkisini artırma potansiyeline sahiptir. Analiz edilen çalışmaların duyulara hitap etme durumları Tablo 'deki gibidir.

Tablo 1. Duyulara Hitap Etme (Addressing the Senses)

\begin{tabular}{lccc}
\multicolumn{4}{c}{ (Addressing the Senses) } \\
\hline & Evet & Hayır & Bilgi yok \\
Görme duyusu & 30 & 0 & 0 \\
İşitme duyusu & 15 & 6 & 9 \\
Dokunma duyusu & 14 & 15 & 1 \\
Koklama duyusu & 0 & 23 & 7 \\
\hline
\end{tabular}

Gerçekleştirilen SG uygulamaları daldırma düzeyleri açısından değerlendirildiğinde; sadece görme duyusuna hitap ettiği tespit edilen 6 çalışma (\%20), sadece görme ve işitme duyularına hitap eden 10 çalışma (\%33) ile sadece görme ve dokunma hitap eden 1 çalışma (\%3) yarl daldırıcı SG uygulaması olarak değerlendirilmiştir (Tablo 2). Görme, işitme ve dokunma duyularının her üçüne hitap eden 5 çalışma (\%17) ile sadece görme ve dokunma duyularına hitap ettiği halde sunduğu dokunsal cihazlarla gerçek cerrahi operasyonlara çok yakın sistemlerin kullanıldığı 8 çalışma (\%27) tam daldırıcı SG uygulaması olarak değerlendirilmiştir (Tablo 2).

\section{SONUC (CONCLUSION)}

$\mathrm{Bu}$ çalışmada, SG uygulamalarıyla eğitim amaçlı gerçekleştirilen çalışmalarda kullanılan cihazlar, daldırma etkileri (bireyin sanal gerçeklik cihazıyla/cihazlarıyla fiziki ortamla olan bağının belirli sınırlılıklarla kesilerek sanal ortamda bulunma hissinin yaratılması) yönünden ele alınmış, yeterlilikleri ve sundukları fırsatlar bakımından incelenmiştir.

İncelenen çalışmaların önemli bir bölümünün sağlık alanında yapılan eğitim uygulamalarını (\%53) içerdiği tespit edilmiştir. Çalışmaların tamamında SG'nin doğası gereği görme duyusuyla, \%50'sinde işitme ve \%47'sinde dokunma duyusuyla etkileşim içeren cihazlar kullanıldığı görülmüş, koku duyusuna hitap eden çalışmaya ise rastlanmamıştır. Heilig tarafindan 1962 yılında tarafından geliştirilen Sensorama'da [86] motosiklet kullanan kişinin kendisini Brooklyn sokaklarında hissetmesi için restoran kokuları kullanılmış, birden fazla duyuya hitap edilerek katılımcının kendisini oluşturulan sanal dünyada hissetmesi amaçlanmıştır. Dış dünyayı doğrudan beyne bağlaması açısından ve dikkat, reaksiyon süresi ve duygusal durum ile doğrudan ilişkili olmasından dolayı koku duyusunun SG ortamlarında kullanılması daldırma ve uzakta var olma hislerini arttırma potansiyeline sahiptir [46]. Analiz edilen çalışmalara bakıldığında koku duyusuna hitap eden çalışmanın bulunmaması, çalışmaların sadece yaklaşık yarısında işitme ve dokunma duyularına hitap edilmesi göz önüne alınarak eğitim alanında kullanılan SG uygulamalarının daldırma etkisi açısından çok güçlü olmadıkları söylenebilir. Analiz edilen $12(\% 40)$ çalışmada ise katılımcıların uygulama esnasında serbest hareket etme imkanına sahip olduğu görülmüştür. Daldırma etkisi açısından kullanıcılara serbest hareket/yürüme özgürlüğü tanınması önemlidir.

Kullanılan cihaz türü bakımından, çalışmaların \%67'sinde SG gözlüklerinin (Head Mounted Display - HMD) tercih edildiği, \%10'unda ise sanal mağaraların (CAVE) kullanıldığı görülmüştür. Kurulum ve maliyet faktörleri göz önüne alındığında CAVE kullanılan çalışma sayısının HMD kullanılan çalışma sayısından düşük olmasının doğal olduğu çıkarımı yapılabilir. Çalışmalarda en fazla kullanılan HMD cihazının ise Oculus Rift olduğu görülmüştür. Söz konusu SG cihazı ve çalışmada kullanılan birçok HMD cihazı görme, işitme, dokunarak sanal ortam ile etkileşim kurma ve serbest hareket etme olanaklarını sunarak daldırma düzeyi açısından önemli avantajlar sunmaktadır. Buna karşın incelenen çalışmaların bu avantajların kullanılmadığı görülmektedir. $\mathrm{Bu}$ ise, eğitim alanında kullanılan bazı SG uygulamalarında; daldırma düzeyinin, teknolojik imkânlara rağmen, yeterince etkin kullanılmadığını göstermektedir.

Birden fazla duyuya hitap etme, HMD'ler ve dokunsal ekipmanlarla kullanıcıya sanal dünya ile etkileşime girme gibi olanaklar yönünden incelendiğinde tam daldırıcı $S G$ uygulaması kullanılan çalışma sayısının (\%57), yarı daldırıcı SG uygulaması kullanılan çalışma sayısından (\%43) düşük olması günümüzün teknolojik imkânlarının yeterince kullanılmadığının farklı bir göstergesidir. 
Hareket izleme sensörleri sanal gerçeklik uygulamalarında nicel verilerin toplanmasını kolaylaştırarak araştırma için somut veriler elde edilmesine olanak tanımakta, ayrıca kullanıcının bazı el ve vücut hareketlerini sanal ortamdaki avatara aktararak daldırma düzeyini arttırmaktadır. İncelenen çalışmaların yaklaşık yarısında (\%53) hareket izleme sensörleri kullanıldığı tespit edilmiştir. Eğitim amacıyla kullanılan SG uygulamalarında daldırma düzeylerinin daha iyi seviyelere çıkarılması açısından, çalışmalardaki hareket izleme sensörü kullanım oranının arttırılması gerektiği söylenebilir.

Son yıllarda akıllı telefon kullanımının artması ve sanal gerçeklik uygulamalarının bu cihazlarla kullanımının kolay olması nedeniyle yapılan çalışmalarda akıllı telefon kullanımının arttığı söylenebilir. Buna paralel olarak eğitim alanında daldırma düzeyini arttırabilecek cihazlar ile yapılan SG uygulamalarının sayısının da son yıllarda artış gösterdiği görülmektedir. Google Cardboard gibi oldukça düşük maliyetli ürünler akıllı telefonlar kullanılarak bir HMD'ye olarak kullanılabilmektedir. Bu ise eğitim için geliştirilen akıllı telefon uyumlu SG uygulamalarının, kolay ulaşılabilirlik açısından oldukça değerli olduğunu göstermektedir.

SG teknolojilerini simülasyonlardan ve klasik üç boyutlu sanal ortamlardan farklılaştıran temel özelliklerden birisi daldırma etkisidir. Simülasyonlarda ve klasik üç boyutlu sanal ortamlarda kullanıcı sanal dünyaya adapte olmaktadır; fakat çoğu zaman kendini sanal dünyanın bir parçası olarak hissetmemektedir. SG deneyiminde ise katılımcı yüksek oranda uzakta (sanal ortamda) var olma hissi yaşamaktadır. $\mathrm{Bu}$ farklılık SG'nin özünü oluşturmaktadır. Bundan dolayı yapılan veya yapılacak çalışmalarda daldırma etkisinin yeterli düzeyde tutulması oldukça önemlidir.

Eğitim alanında SG uygulamalarının kullanımında önemli bir efektif yaklaşım ortaya çıkmaktadır: yaparak yaşayarak öğrenme... Özünde öğrencinin öğrenme sürecinde aktif rol alarak öğrenmesini barındıran yaparak yaşayarak öğrenme yaklaşımı ve SG teknolojilerinin sunduğu özellikler birbirini tamamlamaktadır. SG uygulamalarının kullanımıyla, öğrenci gerçek hayatta deneyimleme şansının düşük veya imkânsız olduğu yaşam durumlarında, yaparak yaşayarak öğrenme firsatını yakalamaktadır. Üstelik daldırma düzeyinin yeterli ölçüde olması ile bu deneyim gerçek hayattakine yakın olabilecek derecede, yüksek oranda uzakta var olma hissi ile gerçekleşmektedir.

Elde edilen bulgulara bakıldığında, eğitim alanında sanal gerçeklik uygulamalarının kullanıldığ daldırma etkisinin beklenilen ölçüde etkili olarak kullanılmadığı görülmektedir. Birden fazla duyuya hitap etme, yüksek daldırma etkisine sahip olma ve hareket izleme sensörleri aracılığıyla katılımcının hareketlerini sanal ortama aktarma gibi yönlerden donanımlı cihazların sayısının son yıllarda artış gösterdiği söylenebilir. Son yıllarda yapılan çalışmaların bu artıştan etkilendiği, buna rağmen daldırma etkisi yüksek çalışmaların sayısının yeterli düzeyde olmadığı savunulabilir.

Birden fazla duyuya hitap eden, kullanıcılara sanal dünyada yürüyormuş gibi serbest hareket etme şansı sunan, hareket izleme sensörleri ile kullanıcının hareket, jest ve mimiklerinin sanal ortama aktaran, özetle tam daldırıcı etkiye sahip SG uygulamalarının, teknolojinin sunduğu firsatlar ile daha fazla kullanılabileceği söylenebilir. Yapılan bu çalışmanın, SG alanında yapılan çalışmalarda kullanılacak cihazların daldırma yönlerine dikkat çekmesi açısından gelecekteki çalışmalara 1 şı tutması beklenmektedir.

\section{KAYNAKLAR (REFERENCES)}

[1] D. A. Guttentag, "Virtual reality: Applications and implications for tourism”, Tourism Management, 31(5), 637-651, 2010.

[2] K. Schwienhorst, "The State of VR: A Meta-Analysis of Virtual Reality Tools in Second Language Acquisition", Computer Assisted Language Learning, 15(3), 221-239, 2002.

[3] R. Blach, "Virtual Reality Technology - An Overview", Product Engineering, Springer, Dordrecht, 21-64, 2008.

[4] L. Freina, M. Ott, "A Literature Review on Immersive Virtual Reality in Education: State Of The Art and Perspectives", Proceedings of eLearning and So ware for Education (eLSE), Romanya, 1, 133-141, 2015.

[5] C. Tuncer, İ. Şimşek, "Eğitimde Yeni Teknolojiler: Sanal Gerçeklik”, Eğitim Teknolojileri Okumaları, Editörler: A. İşman, H. F. Odabaşı, B. Akkoyunlu, Ankara: TOJET - The Turkish Online Journal of Educational Technology, 351-362, 2016

[6] C. Anthes, R. J. García-Hernández, M. Wiedemann, D. Kranzlmüller, "State of the art of virtual reality technology", 2016 IEEE Aerospace Conference, Big Sky, Montana, United States, $1-19,2016$

[7] B. Çavaş, P. Huyugüzel Çavaş, B. T. Can, "Eğitimde Sanal Gerçeklik", TOJET: The Turkish Online Journal of Educational Technology, 3(4), 110-116, 2004.

[8] M. J. Kim, X. Wang, P. E. D. Love, H. Li, S.-C. Kang, "Virtual reality for the built environment: A critical review of recent advances", Journal of Information Technology in Construction, 18, 279-305, 2013.

[9] D. Pasco, "The Potential of Using Virtual Reality Technology in Physical Activity Settings", Quest, 65(4), 429-441, 2013.

[10] D. Passig, "Revisiting the Flynn Effect through 3D Immersive Virtual Reality (IVR)", Computers \& Education, 88, 327-342, 2015 .

[11] M. E. Portman, A. Natapov, D. Fisher-Gewirtzman, "To go where no man has gone before: Virtual reality in architecture, landscape architecture and environmental planning", Computers, Environment and Urban Systems, 54(Supplement C), 376-384, 2015 .

[12] L.-X. Pan, "The application of virtual reality technology to digital tourism systems", International Journal of Simulation: Systems, Science and Technology, 17(18), 2.1-2.5, 2016. 
[13] L. T. Ritz, A. R. Buss, "A Framework for Aligning Instructional Design Strategies with Affordances of CAVE Immersive Virtual Reality Systems", TechTrends, 60(6), 549-556, 2016.

[14] K. McMillan, K. Flood, R. Glaeser, "Virtual reality, augmented reality, mixed reality, and the marine conservation movement", Aquatic Conservation: Marine and Freshwater Ecosystems, 27(S1), 162-168, 2017.

[15] T. D. Parsons et al., "Virtual reality in pediatric psychology", Pediatrics, 140, S86-S91, 2017.

[16] C. Ferguson, P. M. Davidson, P. J. Scott, D. Jackson, L. D. Hickman, "Augmented reality, virtual reality and gaming: an integral part of nursing”, Contemp Nurse, 51(1), 1-4, 2015.

[17] M. Liu, M. Curet, "A Review of Training Research and Virtual Reality Simulators for the da Vinci Surgical System", Teaching and Learning in Medicine, 27(1), 12-26, 2015.

[18] J. Olasky et al., "Identifying Opportunities for Virtual Reality Simulation in Surgical Education: A Review of the Proceedings from the Innovation, Design, and Emerging Alliances in Surgery (IDEAS) Conference: VR Surgery", Surgical Innovation, 22(5), 514-521, 2015.

[19] P. Piromchai, A. Avery, M. Laopaiboon, G. Kennedy, S O'Leary, "Virtual reality training for improving the skills needed for performing surgery of the ear, nose or throat", The Cochrane database of systematic reviews, 2015(9), 2015.

[20] N. Rudarakanchana, H. Van, L. Desender, N. J. Cheshire, "Virtual reality simulation for the optimization of endovascular procedures: Current perspectives", Vascular Health and Risk Management, 11, 195-202, 2015.

[21] G. S. Ruthenbeck, K. J. Reynolds, "Virtual reality for medical training: The state-of-the-art", Journal of Simulation, 9(1), 16-26, 2015 .

[22] F. Aïm, G. Lonjon, D. Hannouche, R. Nizard, "Effectiveness of Virtual Reality Training in Orthopaedic Surgery", Arthroscopy Journal of Arthroscopic and Related Surgery, 32(1), 224-232, 2016.

[23] M. Alaker, G. R. Wynn, T. Arulampalam, "Virtual reality training in laparoscopic surgery: A systematic review \& meta-analysis", International Journal of Surgery, 29, 85-94, 2016.

[24] J. D. Bric, D. C. Lumbard, M. J. Frelich, J. C. Gould, "Current state of virtual reality simulation in robotic surgery training: a review", Surgical Endoscopy and Other Interventional Techniques, 30(6), 2169-2178, 2016.

[25] A. Moglia, V. Ferrari, L. Morelli, M. Ferrari, F. Mosca, A. Cuschieri, "A Systematic Review of Virtual Reality Simulators for Robot-assisted Surgery", European Urology, 69(6), 1065 1080, 2016.

[26] S. Parsons, "Authenticity in Virtual Reality for assessment and intervention in autism: A conceptual review", Educational Research Review, 19(Supplement C), 138-157, 2016.

[27] N. Saxena et al., "Virtual reality environments for health professional education", Cochrane Database of Systematic Reviews, 2016(2), 2016.

[28] N. Vaughan, V. N. Dubey, T. W. Wainwright, R. G. Middleton, "A review of virtual reality based training simulators for orthopaedic surgery", Medical Engineering and Physics, 38(2), 59-71, 2016.
[29] A. Bashiri, M. Ghazisaeedi, L. Shahmorasdi, "The opportunities of virtual reality in the rehabilitation of children with attention deficit hyperactivity disorder: A literature review", Korean Journal of Pediatrics, 60(11), 337-343, 2017.

[30] K. Iamsakul, A. Pavlovcik, J. Calderon, L. Sanderson, "PROJECT HEAVEN: Preoperative training in virtual reality", Surgical Neurology International, 8(1), 2017.

[31] J. T. Lui, M. Y. Hoy, "Evaluating the Effect of Virtual Reality Temporal Bone Simulation on Mastoidectomy Performance: A Meta-analysis”, Otolaryngology-Head and Neck Surgery, 156(6), 1018-1024, 2017.

[32] M. Pfandler, M. Lazarovici, P. Stefan, P. Wucherer, M. Weigl, "Virtual reality-based simulators for spine surgery: a systematic review”, Spine Journal, 17(9), 1352-1363, 2017.

[33] S. G. Kang et al., "An Effective Repetitive Training Schedule to Achieve Skill Proficiency Using a Novel Robotic Virtual Reality Simulator", Journal of Surgical Education, 72(3), 369-376, 2015.

[34] G. Sankaranarayanan et al., "Face and construct validation of a next generation virtual reality (Gen2-VR@) surgical simulator", Surgical Endoscopy and Other Interventional Techniques, 30(3), 979-985, 2016.

[35] A. Winkler-Schwartz et al., "Bimanual Psychomotor Performance in Neurosurgical Resident Applicants Assessed Using NeuroTouch, a Virtual Reality Simulator", Journal of Surgical Education, 73(6), 942-953, 2016.

[36] P. Harrison et al., "The Validation of a Novel Robot-Assisted Radical Prostatectomy Virtual Reality Module", Journal of Surgical Education, 75(3), 758-766, 2018.

[37] C. Repetto, B. Colombo, G. Riva, "Is Motor Simulation Involved During Foreign Language Learning? A Virtual Reality Experiment", SAGE Open, 5(4), 2015.

[38] A. Grabowski, J. Jankowski, "Virtual Reality-based pilot training for underground coal miners", Safety Science, 72(Supplement C), 310-314, 2015.

[39] D. C. Schwebel, T. Combs, D. Rodriguez, J. Severson, V. Sisiopiku, "Community-based pedestrian safety training in virtual reality: A pragmatic trial", Accident Analysis \& Prevention, 86(Supplement C), 9-15, 2016.

[40] E. K. Orman, H. E. Price, C. R. Russell, "Feasibility of Using an Augmented Immersive Virtual Reality Learning Environment to Enhance Music Conducting Skills", Journal of Music Teacher Education, 27(1), 24-35, 2017.

[41] M. Kolomaznik, M. Sullivan, K. Vyvyan, "Can virtual reality engage students with teamwork?", International Journal of Innovation in Science and Mathematics Education, 25(4), 32-44, 2017.

[42] M. Roussou, "Immersive Interactive Virtual Reality in the Museum", i3 Spring Days 2000 - Workshop on Interactive Learning Environments for Children, Yunanistan, 2000.

[43] J. Psotka, "Immersive training systems: Virtual reality and education and training”, Instructional Science, 23(5-6), 405-431, 1995.

[44] H. G. Hoffman et al., "Manipulating presence influences the magnitude of virtual reality analgesia", Pain, 111(1), 162-168, 2004. 
[45] Ü. Çakiroğlu, S. Gökoğlu, "Development of fire safety behavioral skills via virtual reality", Computers \& Education, 133, 56-68, 2019.

[46] M. A. A. Gutiérrez, F. Vexo, D. Thalmann, Stepping into Virtual Reality, London: Springer London, 2008.

[47] O. Bamodu, X. Ye, "Virtual Reality and Virtual Reality System Components", Proceedings of the 2nd International Conference On Systems Engineering and Modeling, Paris, France, 921-924, 2013

[48] N. Magnenat-Thalmann, U. Bonanni, "Haptics in Virtual Reality and Multimedia", IEEE Multimedia, 13(3), 6-11, 2006.

[49] A. Lecuyer, "Playing with Senses in VR: Alternate Perceptions Combining Vision and Touch", IEEE Computer Graphics and Applications, 37(1), 20-26, 2017.

[50] M. Limniou, D. Roberts, N. Papadopoulos, "Full immersive virtual environment CAVETM in chemistry education", Computers \& Education, 51(2), 584-593, 2008.

[51] C. A. Kilmon, L. Brown, S. Ghosh, A. Mikitiuk, "Immersive virtual reality simulations in nursing education", Nursing Education Perspectives, 31(5), 314-317, 2010.

[52] F. Gutierrez et al., "The Effect of Degree of Immersion upon Learning Performance in Virtual Reality Simulations for Medica Education", Studies in Health Technology and Informatics, 125, $155-160,2007$.

[53] R. L. Jackson, E. Fagan, "Collaboration and learning within immersive virtual reality", Proceedings of the third international conference on Collaborative virtual environments, 83-92, 2000.

[54] D. A. Bowman, R. P. McMahan, "Virtual Reality: How Much Immersion Is Enough?”, Computer, 40(7), 36-43, 2007.

[55] M.-L. Ryan, "Immersion vs. Interactivity: Virtual Reality and Literary Theory”, SubStance, 28(2), 110-137, 1999.

[56] L. Cohen, L. Manion, K. Morrison, Research Methods in Education, 6th ed. New York, ABD: Routledge, 2007.

[57] J. R. Fraenkel, N. E. Wallen, How to evaluate \& design research in education, 4th ed. New York, ABD: McGraw-Hill, 2000.

[58] W. Bauer, "Introduction", Educational Philosophy and Theory, 35(2), 133-137, 2003.

[59] K. Bekelis, D. Calnan, N. Simmons, T. A. Mackenzie, G. Kakoulides, "Effect of an immersive preoperative virtual reality experience on patient reported outcomes: A randomized controlled trial", Annals of Surgery, 265(6), 1068-1073, 2017.

[60] C. Carbonell-Carrera, J. L. Saorín, "Geospatial Google Street View with virtual reality: Amotivational approach for spatial training education", ISPRS International Journal of GeoInformation, 6(9), 2017.

[61] F. Górski, P. Bun, R. Wichniarek, P. Zawadzki, A. Hamrol, "Effective design of educational virtual reality applications for medicine using knowledge-engineering techniques", Eurasia Journal of Mathematics, Science and Technology Education, 13(2), 395-416, 2017.

[62] O. Halabi, S. A. El-Seoud, J. M. Aljaam, H. Alpona, M. AlHemadi, D. Al-Hassan, "Design of immersive virtual reality system to improve communication skills in individuals with autism", International Journal of Emerging Technologies in Learning, 12(5), 50-64, 2017.

[63] O. Ossmy, R. Mukamel, "Using virtual reality to transfer motor skill knowledge from one hand to another", Journal of Visualized Experiments, 2017(127), 2017.

[64] J. Pirker, I. Lesjak, C. Gütl, "An educational physics laboratory in mobile versus room scale virtual reality - A comparative study", International Journal of Online Engineering, 13(8), 106-120, 2017.

[65] F. J. Real et al., "A Virtual Reality Curriculum for Pediatric Residents Decreases Rates of Influenza Vaccine Refusal", Academic Pediatrics, 17(4), 431-435, 2017.

[66] M. Ros, J.-V. Trives, N. Lonjon, "From stereoscopic recording to virtual reality headsets: Designing a new way to learn surgery", Neurochirurgie, 63(1), 1-5, 2017

[67] A. S. Wilson, J. O’Connor, L. Taylor, D. Carruthers, "A 3D virtual reality ophthalmoscopy trainer", Clinical Teacher, 14(6), 427-431, 2017.

[68] W. S. Alhalabi, "Virtual reality systems enhance students' achievements in engineering education", Behaviour and Information Technology, 35(11), 919-925, 2016.

[69] E. Madrigal, S. Prajapati, J. C. Hernandez-Prera, "Introducing a virtual reality experience in anatomic pathology education", American Journal of Clinical Pathology, 146(4), 462-468, 2016.

[70] D. Passig, D. Tzuriel, G. Eshel-Kedmi, "Improving children's cognitive modifiability by dynamic assessment in 3D Immersive Virtual Reality environments", Computers \& Education, 95(Supplement C), 296-308, 2016.

[71] T. M. Takala, L. Malmi, R. Pugliese, T. Takala, "Empowering Students to Create Better Virtual Reality Applications: A Longitudinal Study of a VR Capstone Course", Informatics in Education, 15(2), 287-317, 2016.

[72] J. S. Cho et al., "Virtual reality training improves da vinci performance: A prospective trial", Journal of Laparoendoscopic and Advanced Surgical Techniques, 23(12), 992-998, 2013.

[73] P. P. Gomez, R. E. Willis, S. Van, "Development of a virtual reality robotic surgical curriculum using the da Vinci Si surgical system", Surgical Endoscopy and Other Interventional Techniques, 29(8), 2171-2179, 2015.

[74] D. J. Kiely et al., "Virtual reality robotic surgery simulation curriculum to teach robotic suturing: a randomized controlled trial", Journal of Robotic Surgery, 9(3), 179-186, 2015.

[75] M. A. Liss, C. J. Kane, T. Chen, J. Baumgartner, I. H. Derweesh, "Virtual reality suturing task as an objective test for robotic experience assessment”, BMC Urology, 15(1), 2015.

[76] M. Valdis, M. W. A. Chu, C. M. Schlachta, B. Kiaii, "Validation of a novel virtual reality training curriculum for robotic cardiac surgery a randomized trial", Innovations: Technology and Techniques in Cardiothoracic and Vascular Surgery, 10(6), 383$388,2015$.

[77] V. Mouraviev et al., "Urology residents experience comparable workload profiles when performing live porcine nephrectomies and robotic surgery virtual reality training modules", Journal of Robotic Surgery, 10(1), 49-56, 2016.

[78] K. Kilteni, I. Bergstrom, M. Slater, "Drumming in immersive 
virtual reality: The body shapes the way we play", IEEE Transactions on Visualization and Computer Graphics, 19(4), 597-605, 2013.

[79] T. D. Parsons, C. G. Courtney, M. E. Dawson, "Virtual reality Stroop task for assessment of supervisory attentional processing", Journal of Clinical and Experimental Neuropsychology, 35(8), 812-826, 2013.

[80] B. Patrão, P. Menezes, "A virtual reality system for training operators", International Journal of Online Engineering, 9(Special issue 8), 53-55, 2013.

[81] S. Quero, M. Á. Pérez-Ara, J. Bretón-López, A. García-Palacios, R. M. Baños, C. Botella, "Acceptability of Virtual Reality Interoceptive Exposure for the Treatment of Panic Disorder with Agoraphobia", British Journal of Guidance \& Counselling, 42(2), 123-137, 2014.

[82] D. Freeman, N. Evans, R. Lister, A. Antley, G. Dunn, M. Slater, "Height, social comparison, and paranoia: An immersive virtual reality experimental study", Psychiatry Research, 218(3), 348-
352,2014

[83] X. Gong, Y. Liu, Y. Jiao, B. Wang, J. Zhou, H. Yu, "A novel earthquake education system based on virtual reality", IEICE Transactions on Information and Systems, E98D(12), 2242-2249, 2015.

[84] J. Gutiérrez-Maldonado, M. Ferrer-García, J. Pla-Sanjuanelo, A. Andrés-Pueyo, A. Talarn-Caparrós, "Virtual reality to train diagnostic skills in eating disorders. Comparison of two low cost systems", Annual Review of CyberTherapy and Telemedicine, 13, 75-81, 2015.

[85] M. Norrby, C. Grebner, J. Eriksson, J. Boström, "Molecular Rift: Virtual Reality for Drug Designers", Journal of Chemical Information and Modeling, 55(11), 2475-2484, 2015.

[86] M. L. Heilig, "Sensorama simulator", 1962.

[87] C. Carbonell-Carrera, J. L. Saorín, "Geospatial Google Street View with virtual reality: Amotivational approach for spatial training education", ISPRS International Journal of GeoInformation, 6(9), 2017. 
Tablo 2. Calıșma Kapsamında İncelenen Makaleler

(Analysed Studies)

\begin{tabular}{|c|c|c|c|c|c|c|c|c|c|c|c|}
\hline$\frac{\mathbb{\Xi}}{\stackrel{\mathbb{E}}{\Xi}}$ & 矛 & 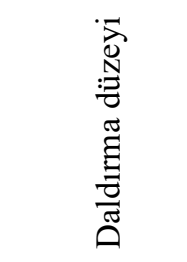 & 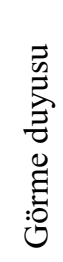 & 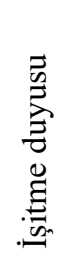 & 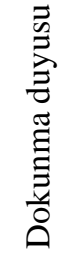 & 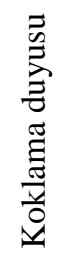 & 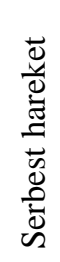 & 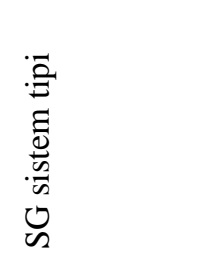 & 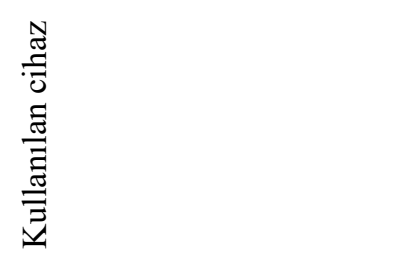 & 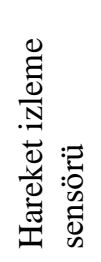 & 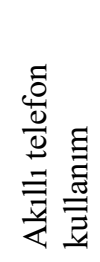 \\
\hline [59] & Ameliyat öncesi canlandırma & yarı daldırıcı & $\checkmark$ & $\checkmark$ & $x$ & $x$ & & HMD & Oculus Rift & $x$ & $x$ \\
\hline [87] & Mekansal Eğitim & yarı daldırıcı & $\checkmark$ & $x$ & $\checkmark$ & $x$ & & HMD & Woxter Neo Virtual Reality & $x$ & $\checkmark$ \\
\hline [61] & Cerrahi Eğitimi & tam daldirlcl & $\checkmark$ & $x$ & $\checkmark$ & $x$ & & HMD & Samsung Gear VR & $\checkmark$ & $\checkmark$ \\
\hline [62] & İletişim Becerilerini Arttırmak & yarı daldırıcı & $\checkmark$ & $\checkmark$ & $x$ & $x$ & $\checkmark$ & CAVE+HMD & Oculus Rift & $\checkmark$ & $x$ \\
\hline [40] & Müzik Becerileri Arttırma & yarı daldırıcı & $\checkmark$ & $\checkmark$ & $x$ & $x$ & $x$ & HMD & Vuzix Wrap 1200VR & $\checkmark$ & $x$ \\
\hline [63] & Motor becerileri Eğitimi & yart daldırıcı & $\checkmark$ & $x$ & $x$ & $x$ & $\checkmark$ & HMD & & $\checkmark$ & \\
\hline [64] & Eğitim ortamı tasarımı & tam daldırıcı & $\checkmark$ & $\checkmark$ & $\checkmark$ & $x$ & $x$ & HMD & HTC Vive+Samsung Gear VR & $\checkmark$ & $\checkmark$ \\
\hline [65] & Inf. Aşlsı reddetme & yarı daldırıcı & $\checkmark$ & $\checkmark$ & $x$ & $x$ & $x$ & HMD & Oculus Rift & $x$ & $x$ \\
\hline [66] & Cerrahi eğitimi & yarı daldırıcı & $\checkmark$ & $x$ & $x$ & $x$ & $x$ & HMD & Oculus Rift & $x$ & $x$ \\
\hline [67] & Göz Muayenesi Eğitimi & yarı daldırıcı & $\checkmark$ & $x$ & $x$ & $x$ & & HMD & RITECH II Google Cardboard & $x$ & $\checkmark$ \\
\hline [68] & Mühendislik Eğitimi & yarı daldırıcı & $\checkmark$ & & & $x$ & $\checkmark$ & CAVE+HMD & Oculus Rift & $\checkmark$ & $x$ \\
\hline [69] & Anatomik Patoloji & tam daldiricı & $\checkmark$ & $\checkmark$ & $\checkmark$ & $x$ & & HMD & & $x$ & $\checkmark$ \\
\hline [77] & Üroloji Robotik cerrahi & tam daldırıcı & $\checkmark$ & & $\checkmark$ & & $x$ & dV-Trainer & & $x$ & \\
\hline [70] & Bilişsel Gelişim & yarı daldırıcı & $\checkmark$ & $x$ & $x$ & $x$ & $x$ & HMD & & $x$ & $x$ \\
\hline [34] & Cerrahi Eğitimi & tam daldırıcı & $\checkmark$ & $\checkmark$ & $\checkmark$ & $x$ & $x$ & HMD & VR2000 + Oculus Rift + Zsight & $\checkmark$ & \\
\hline [71] & Capstone / VR Dersi & tam daldirlci & $\checkmark$ & $\checkmark$ & $\checkmark$ & $x$ & $\checkmark$ & CAVE+HMD & Oculus Rift & $\checkmark$ & $x$ \\
\hline [72] & Robotik cerrahi & tam daldiricı & $\checkmark$ & & $\checkmark$ & & $x$ & dV-Trainer & & $x$ & \\
\hline [78] & SG Ortamında Bedensel Farkındalık & tam daldirlci & $\checkmark$ & $\checkmark$ & $\checkmark$ & $x$ & $\checkmark$ & HMD & NVISOR SX111 & $\checkmark$ & $x$ \\
\hline [79] & Denetleyici Dikkat İsleme & yarl daldırıcı & $\checkmark$ & $\checkmark$ & $x$ & $x$ & $\checkmark$ & HMD & eMagin Z800 & $\checkmark$ & $x$ \\
\hline [80] & Vinç Operatör Eğitimi & yarı daldırıce & $\checkmark$ & $\checkmark$ & $x$ & $x$ & $\checkmark$ & HMD & eMagin Z800 & $\checkmark$ & $x$ \\
\hline [81] & Panik Bozukluğu Tedavisi & yart daldırıcı & $\checkmark$ & $\checkmark$ & $x$ & $x$ & $\checkmark$ & HMD & V6 Head Mount Display & $\checkmark$ & $x$ \\
\hline [82] & Sosyal Kabullenme & yarı daldırıcı & $\checkmark$ & $\checkmark$ & $x$ & $x$ & $\checkmark$ & HMD & NVISOR SX111 & $\checkmark$ & $x$ \\
\hline [73] & Robotik cerrahi & tam daldırıcı & $\checkmark$ & & $\checkmark$ & & $x$ & $\begin{array}{l}\text { da Vinci Skills } \\
\text { Simulator }\end{array}$ & & $x$ & \\
\hline [83] & Deprem Ĕ̈itimi & yarı daldırıcı & $\checkmark$ & $\checkmark$ & $x$ & $x$ & $\checkmark$ & HMD & VUZIX Wrap 1200 & $\checkmark$ & $x$ \\
\hline [84] & Beslenme Bozukluğu Tedavisi & yarl daldirlct & $\checkmark$ & $\checkmark$ & $x$ & $x$ & $\checkmark$ & HMD & Oculus Rift DK1 & $\checkmark$ & $x$ \\
\hline [33] & Robotik cerrahi & tam daldiricı & $\checkmark$ & & $\checkmark$ & & $x$ & dV-Trainer & & $x$ & \\
\hline [74] & Robotik cerrahi & tam daldıricı & $\checkmark$ & & $\checkmark$ & & $x$ & $\begin{array}{l}\text { da Vinci Skills } \\
\text { Simulator }\end{array}$ & & $x$ & \\
\hline [75] & Robotik cerrahi & tam daldirlcı & $\checkmark$ & & $\checkmark$ & & $x$ & $\begin{array}{l}\text { da Vinci Skills } \\
\text { Simulator }\end{array}$ & & $x$ & \\
\hline [85] & Kimya Eğitimi & yart daldırıct & $\checkmark$ & & $x$ & $x$ & $\checkmark$ & HMD & Oculus Rift DK2 & $\checkmark$ & $x$ \\
\hline [76] & Robotik cerrahi & tam daldırıcı & $\checkmark$ & & $\checkmark$ & & $x$ & $\begin{array}{l}\text { da Vinci Skills } \\
\text { Simulator }\end{array}$ & & $x$ & \\
\hline
\end{tabular}

